\title{
Anticraving and Beyond - Innovative Therapieansätze in der Suchtbehandlung
}

Im Bereich der Suchtherapie gibt es je nach Art der Drogen unterschiedliche Therapieansätze. Während bei Patienten mit Alkohol- und Tabakabhängigkeit nach wie vor die Abstinenz als das sicherste Therapieziel gilt, wird bei Opiatabhängigkeit die Substitutionstherapie meist einer Abstinenzorientierung vorgezogen, da es bei abstinenten Opiatabhängigen im Rückfall zu tödlichen Überdosierungen kommen kann. Im Bereich der Alkoholabhängigkeit gibt es Ansätze zur Konsumreduktion, die nicht auf Abstinenz zielen, insbesondere bei Patienten, die keine schwere Alkoholabhängigkeit mit beispielsweise lebensbedrohlichen Entzugserscheinungen aufweisen, sowie als Therapieansatz für Patienten, die sich derzeit keine Alkoholabstinenz vorstellen können, von einer Konsumreduktion aber profitieren [1]. Bezüglich aller Suchterkrankungen gilt die Betonung der psychosozialen Versorgung, die neben dem Fokus auf die somatisch schädlichen Wirkungen der Droge die sozialen Folgen im Sinne von Verschuldung oder Arbeitsplatzverlust ebenso berücksichtigt wie zwischenmenschliche Konflikte, und die die Motivation zur Verhaltensänderung durch entsprechende psychotherapeutische Interventionen stützt [2]

Bezüglich der medikamentösen Behandlung der Alkoholabhängigkeit sind in Deutschland Naltrexon, Nalmefene und Acamprosat zugelassen. Naltrexon und Acamprosat dienen der Aufrechterhaltung der Alkoholabstinenz, wobei Naltrexon mu-Opiatrezeptoren blockiert und damit die positiv verstärkende Wirkung des Alkoholkonsums reduzieren soll. Acamprosat interagiert demgegenüber mit glutamatergen NMDA-Rezeptoren und könnte so ein Ungleichgewicht zwischen erregenden und hemmenden Botenstoffen nach Entgiftung positiv beeinflussen. Nalmefene wirkt ähnlich wie Naltrexon, ist aber zur Konsumreduktion bei schädlichem Alkoholkonsum und nicht allzu ausgeprägten Formen der Alkoholabhängigkeit zugelassen [2].

In den letzten Jahrzehnten hat neurobiologische Grundlagenforschung zentrale Me- chanismen der Abhängigkeitserkrankungen identifiziert, die Ziel pharmakologischer und psychotherapeutischer Interventionen sein können. Dazu gehört die Gewöhnung an den Substanzkonsum, der durch Pawlowsche Reize getriggert werden kann, welche in Interaktion mit zielgerichtetem Verhalten eine Tendenz zur Annäherung an Drogenreize und zum Drogenkonsum auslösen können. Gegen diese Tendenz wirkt ein systematisches Verhaltenstraining zur Zurückweisung von Drogenreizen, wie es beispielsweise computerisiert durchgeführt werden kann. Dabei werden für die Drogenreaktion wichtige Hirnareale wie beispielsweise die Aktivierung der Amygdala bei Konfrontation mit Alkoholbildern in ihrer Aktivität herunterreguliert, was wiederrum den Therapieerfolg voraussagt [3]. Bezüglich neuerer Medikamente wurde auf Grund einer Selbstbeschreibung und erster positiver Studien bei schwer alkoholabhängigen Patienten mit Lebererkrankungen der nur gegen Spastik zugelassene GABA-B Agonist Baclofen international diskutiert. Anders als GABA-A Agonisten weist er ein geringes oder gar kein bestehendes $A b$ hängigkeitspotenzial auf und reduziert zentralnervös beispielsweise die Dopamin-Freisetzung, die durch viele Drogen inklusive Alkohol stimuliert wird. Die Studienlage zur Wirkung von Baclofen ist unterschiedlich. Die Substanz passiert die Blut-Hirn-Schranke nur eingeschränkt, sodass gegebenenfalls höhere Dossierungen für zentralnervöse Wirkungen notwendig sind. Tatsächlich beobachtete eine Studie von Müller et al. [4], dass bei individuell flexibler Dosierung, die deutlich über der üblichen Dosierung gegen Spastik lag, Baclofen signifikant besser wirksam war als Placebo, während dies bei anderen Studien nicht der Fall war [5,6]. Weitere interessante Therapieansätze finden sich im Bereich der Antiepileptika, die ebenfalls das Gleichgewicht zwischen erregenden und hemmenden Botenstoffen im akuten Entzug und der frühen Abstinenz modulieren und damit möglicherweise insbesondere bei stress-assoziiertem Alkoholkonsum wirksam sind. Die Wirksamkeit derartiger Substanzen, die über die Beeinflussung des Alkoholverlangens (Craving) hinaus auch die Reaktion auf Alkoholreize beeinflussen können, könnte durch Genotypisierung der betroffenen Individuen verstärkt werden. Dafür gibt es erste Hinweise [7], ein Einsatz dieser Techniken in der klinischen Praxis ist aber noch nicht sinnvoll, da replizierte Befunde weitgehend fehlen.

\section{Interessenkonflikte}

Keine

\section{Autoren}

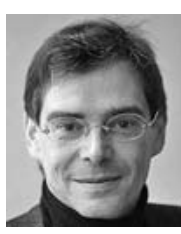

Andreas Heinz ${ }^{1}$, Laura S. Daedelow $^{2}$, Lea Mascarell-Maricic ${ }^{2}$ Annika Rosenthal ${ }^{2}$

1 Direktor Klinik für Psychiatrie und Psychotherapie, Charité - Universitätsmedizin Berlin

2 Klinik für Psychiatrie und Psychotherapie, Charité - Universitätsmedizin Berlin

\section{Korrespondenzadresse}

Prof. Dr. Dr. Andreas Heinz

Direktor

Klinik für Psychiatrie und

Psychotherapie

Charité - Universitätsmedizin Berlin

Charitéplatz 1

10117 Berlin

andreas.heinz@charite.de

\section{Literatur}

[1] Charlet K, Heinz A. Harm reduction - a systematic review on effects of alcohol reduction on physical and mental symptoms. Addict Biol 2016; doi:10.1111/adb.12414

[2] Batra A, Müller CA, Mann K et al. Abhängigkeit und schädlicher Gebrauch von AlkoholDiagnostik und Behandlungsoptionen. Dtsch Arztebl Int 2016; 113: 301-310

[3] Wiers CE, Stelzel C, Gladwin TE et al. Effects of cognitive bias modification training on neural alcohol cue reactivity in alcohol dependence. Am J Psychiatry 2015; 172: 335343

[4] Müller CA, Geisel O, Pelz P et al. High-dose baclofen for the treatment of alcohol dependence (BACLAD study): a randomized, placebo-controlled trial. Eur Neuropsychopharmacol 2015; 25: 1167-1177 
[5] Beraha EM, Salemink E, Goudriaan AE et al. Efficacy and safety of high-dose baclofen for the treatment of alcohol dependence: a multicentre, randomised, double-blind controlled trial. Eur Neuropsychopharmacol 2016; 26: 1950-1959
[6] Garbutt JC, Kampov-Polevoy AB, Gallop R et al. Efficacy and safety of baclofen for alcohol dependence: a randomized, double-blind, placebo-controlled trial. Alcohol Clin Exp Res 2010; 34: 1849-1857
[7] Jorde A, Bach P, Witt SH et al. Genetic variation in the atrial natriuretic peptide transcription factor GATA4 modulates amygdala responsiveness in alcohol dependence. Biol Psychiatry 2014; 75: 790-797 